BULLETIN Bulletin hispanique

HISPANIQUE Université Michel de Montaigne Bordeaux

117-2 | 2015

Métamorphose(s) : représentations et réécritures

\title{
Sur un texte de Diego Rosel
}

à propos de tortues, de métamorphoses et de sensualité (Naples, 1613)

Fernando Copello

\section{(2) OpenEdition}

Journals

Édition électronique

URL : http://journals.openedition.org/bulletinhispanique/4082

DOI : 10.4000/bulletinhispanique.4082

ISSN : 1775-3821

Éditeur

Presses universitaires de Bordeaux

Édition imprimée

Date de publication : 15 décembre 2015

Pagination : 645-654

ISBN : 979-10-300-0041-2

ISSN : 0007-4640

Référence électronique

Fernando Copello, «Sur un texte de Diego Rosel », Bulletin hispanique [En ligne], 117-2 | 2015, mis en

ligne le 15 décembre 2018, consulté le 06 mai 2019. URL : http://journals.openedition.org/

bulletinhispanique/4082 ; DOI : 10.4000/bulletinhispanique.4082 


\title{
Sur un texte de Diego Rosel : à propos de tortues, de métamorphoses et de sensualité (Naples, 1613)
}

\author{
Fernando Copello \\ Université du Maine-Le Mans
}

Un recueil de narrations, publié à Naples en 1613, propose au lecteur des récits étiologiques à caractère comique. Certains de ces textes sont en même temps des métamorphoses. Il sera question dans cet article de la Transformation de la tortue, qui traite de la question de la sensualité. Ces textes de Diego Rosel ont attiré l'attention de Cervantès et de Lope de Vega.

Mots-clés : Diego Rosel y Fuenllana, métamorphose, récit étiologique, Siècle d'Or.

Una colección de narraciones, publicada en Nápoles en 1613, propone al lector relatos etiológicos de carácter cómico. Algunos de estos textos son a la vez metamorfosis. Este artículo se propone estudiar la Transformación del galápago que evoca también el problema de la sensualidad. Estos textos de Diego Rosel llamaron la atención de Cervantes y Lope de Vega en su momento.

Palabras claves: Diego Rosel y Fuenllana, metamorfosis, relato etiológico, Siglo de Oro.

A collection of stories, published in Naples in 1613, provides the reader with etiological comical narratives. Some of these texts contain at the same time metamorphoses. This paper examines the Turtle transformation which also deals with the sensuality issue. Cervantes and Lope de Vega became very interested in these stories by Diego Rosel.

Keywords: Diego Rosel y Fuenllana, Metamorfosis, Etiological narrative, Golden Age. 
$\mathrm{D}$

ANS LE GRAND THÉÂTRE de la nouvelle espagnole du XVII ${ }^{e}$ siècle, qui se joue sur une scène ouverte et prolixe, il y a un arrière-plan. Et c'est dans cet arrière-plan que bouillonnent et s'agitent les idées et les débats qui vont donner naissance au genre de cette novella española dont Cervantès et Lope seront des représentants majeurs, chacun à sa manière, faite d'une simplicité travaillée chez l'un ou d'une érudition tapageuse chez l'autre, chacun à la recherche d'un lecteur que d'autres, comme Juan Pérez de Montalbán, séduisent par le moyen d'intrigues scandaleuses ${ }^{1}$. C'est à partir de cet arrière-plan que je travaille en cherchant des détails pour mieux comprendre l'ensemble.

L'année 1613 est en ce sens une date capitale car l'idéal du genre est loin d'être défini, même s'il est déjà présent sur le marché du livre sous le titre extrêmement significatif de Novelas ejemplares, qui témoigne chez son modeste auteur d'une certaine soif d'infini. Et ce sont les lecteurs, les libraires et les confrères écrivains qui vont valider, vers les années vingt, cette ambition de Miguel de Cervantes. Mais en 1613 le territoire est encore vierge, chacun s'exprime en toute liberté, seuls quelques exemples existent d'une manière possible de tourner en langue castillane cette novella pratiquée dans la lointaine Italie ${ }^{2}$.

L'année 1613 sera donc le point de départ de ces quelques réflexions. C'est à cette date que trois auteurs voués au récit bref proposent leur " produit " dans trois villes différentes, ce qui explique en partie la diffusion inégale de leurs écrits. Cervantès, qu'on connaît mieux, publie ses Nouvelles exemplaires dans la ville de Madrid, redevenue Cour des Espagnes, avant-scène littéraire : il s'agit donc d'un lieu de diffusion extraordinaire. Il est déjà l'auteur du premier Quichotte (1605) qui fait parler de lui. Simultanément, dans un territoire périphérique, ouvert vers la Méditerranée mais considérablement moins important, la ville de Valencia, Sebastián Mey propose un ouvrage hétérogène et illustré, dans une coédition financée par deux libraires et éditée dans la maison d'impression familiale ${ }^{3}$ : le Fabulario. Ce livre, que sa préface destine à un jeune public, n'en est pas moins un champ d'expérimentation du récit bref aux frontières de la fable et de la nouvelle. Encore un détail à retenir : c'est dans les presses de Joan Mey, le grand-père de Sebastián, qu'était paru en 1567 El Patrañuelo

1. Sur la nouvelle espagnole du XVII ${ }^{e}$ siècle, on peut consulter la synthèse d'Isabel Colón Calderón, La novela corta en el siglo XVII, Madrid, Arcadia de Letras, 2001, ainsi que les ouvrages cités. L'introduction de Rafael Bonilla Cerezo à Novelas cortas del siglo XVII, Madrid, Cátedra, 2010, permet d'avoir accès à une bibliographie plus récente. Sur le paratexte dans la nouvelle espagnole, voir Anne Cayuela, Le paratexte au Siècle d'Or. Prose romanesque, livres et lecteurs en Espagne au XVII siècle, Genève, Droz, 1996.

2. Citons les exemples les plus significatifs : le Patrañuelo, recueil de nouvelles de Joan Timoneda publié en 1567, et Noches de invierno d'Antonio de Eslava, publié en 1609.

3. Voir sur cet auteur et sur ce livre : Silvia Monti, "Il Fabulario di Sebastián Mey tra intertestualità e contestualità ", Quaderni di lingue e litterature, $\mathrm{n}^{\circ}$ 14, 1987, p. 133-151; Fernando Copello, "Fiction et jeune public en Espagne au XVII" siècle : le Fabulario de Sebastián Mey ", dans Pierre Civil (sous la dir. de), Écriture, pouvoir et société en Espagne aux XVI et XVII siècles, Paris, Publications de la Sorbonne/Presses de la Sorbonne Nouvelle, 2001, p. 155-169 ; Id. , «À propos de l'illustration des fables : du texte ésopique au Fabulario de Sebastián Mey (1613) ", Le Fablier, ${ }^{\circ} 19,2008$, p. 47-57. 
de Joan Timoneda, livre considéré comme étant le premier recueil espagnol de nouvelles à l'italienne $e^{4}$. Il y a donc une tradition valencienne de la nouvelle en langue castillane, modeste mais certaine. Et pour finir, cet ouvrage de Diego Rosel que je souhaite présenter rapidement pour entamer par la suite l'analyse d'un de ses récits : Parte Primera de varias aplicaciones, y Transformaciones, las cuales tractan, Términos Cortesanos, Práctica Militar, Casos de Estado, en prosa $y$ verso con nuevos Hieroglificos, y algunos puntos morales, publié à Naples chez Juan Domingo Roncallolo en 1613 (mais avec deux textes préliminaires datés à Barcelone en 1607) ${ }^{5}$. Naples est sans doute la plus grande ville espagnole, située à un carrefour géographique considérablement important, mais ce n'est pas dans ce coin de l'autre péninsule qu'on va forger la pratique d'une littérature devenue castillane. Cela explique en partie l'oubli dans lequel sont tombées ces métamorphoses hétéroclites proposées par Diego Rosel, qui n'était pas un Napolitain de souche et dont le texte avait été créé, proposé et partagé pendant les années castillanes de l'auteur, sûrement pendant son séjour à Valladolid, où il a vécu, selon les hypothèses de Williard King ${ }^{6}$. Rosel a été relativement proche de Cervantès, qui lui dédie un sonnet dans les pages préliminaires des Transformaciones (sonnet où il est question d'expérimentation littéraire ${ }^{7}$ ) et il était connu de Lope, qui évoque le livre napolitain dans une de ses nouvelles publiées en $1624:$ « ... Rosel de Fuenllana, un gentilhomme qui se disait porteétendard des parties d'Espagne et qui fit imprimer un livre à Naples [...] tel qu'aucun hypocondriaque ne devrait s'en séparer $»^{8}$.

$S^{\prime}$ il fallait souligner un élément commun à ces trois recueils, je dirais qu'il s'agit d'une même recherche de la variété dans un ensemble qui se veut cohérent. La variété est d'une part l'image la plus fidèle du monde, d'autre part elle est

4. Sur ce point et cette affirmation de Marcelino Menéndez y Pelayo, voir de manière plus générale Augusto Guarino, La narrativa di Joan Timoneda, Napoli, Istituto Universitario Orientale, 1993.

5. Diego Rosel y Fuenllana, Parte Primera de varias aplicaciones, y Transformaciones..., Nápoles, Juan Domingo Roncallolo, 1613 [B. Mazarine : 11310]. Une édition moderne partielle existe : D. Rosel y Fuenllana, Obras selectas, Edición y prólogo de Alan Soons, Chapel Hill, University of North Carolina, coll. "Estudios de Hispanófila ", n 14, 1970. Je cite à partir de l'édition princeps. Juan Domingo Roncallolo est un éditeur napolitain qui s'intéresse, entre autres, au livre en langue castillane. Voir quelques informations à ce propos dans Encarnación Sánchez García, "Imprenta napolitana : los libros del virrey Osuna (1616-1620) ", La Perinola, n 8, 2004, p. 433-461.

6. Diego Rosel aurait été le président d'une academia à l'époque où la Cour se trouvait à Valladolid. Quevedo aurait participé à ces réunions littéraires (Williard King, Prosa novelística y academias literarias en el siglo XVII, Madrid, Real Academia Española, coll. "Anejos del Boletín de la Real Academia Española ", 1963, p. 39-40).

7. Le titre du sonnet est le suivant : «A D. Diego Rosel y Fuenllana inventor de nuevas artes " (D. Rosel, Parte primera..., p. 17).

8. Lope de Vega, Nouvelles à Marcie-Léonarde, Introduction, édition, traduction et notes de Jeanne Agnès et Pierre Guenoun, Paris, Aubier-Montaigne, 1978, p. 255. La nouvelle dont il s'agit est "Guzmán el Bravo ». Par ailleurs, dans le fonds de la librairie d'Alonso Pérez de Montalbán à Madrid se trouvait un exemplaire du livre de Rosel (voir Anne Cayuela, Alonso Pérez de Montalbán. Un librero en el Madrid de los Austrias, Madrid, Calambur, 2005, p. 324). 
la matière même de toute expérimentation. On s'est souvent efforcé de donner une image homogène de l'ensemble des Nouvelles exemplaires de Cervantès en cherchant des ponts et des passerelles entre les manières diverses de narrer chez l'écrivain d'Alcalá. On a pourtant oublié ces vers offerts en guise d'introduction par Fernando Bermúdez y Carvajal qui parle de douze labyrinthes présentant les mille états variés de la nature? ${ }^{9}$. Et rien de plus contrasté, en effet, que la poétique de "La gitanilla », texte qui ouvre le recueil cervantin, et ce dialogue humaniste du "Coloquio de los perros ", la conclusion d'un livre qui se veut ouvert et qui évite la présence d'un cadre narratif structurant. L'examen de la totalité du titre du Fabulario de Sebastián Mey montre encore plus clairement la volonté de créer un livre varié, fait de choses diverses : Fabulario en que se contienen fábulas y cuentos diferentes, algunos nuevos y parte sacados de otros autores. Variété de genres, différence de contes, l'ancien et le nouveau et, encore plus intéressant, cette notion de livre collectif construit à partir de la production de plusieurs auteurs. Nous avons déjà évoqué le long titre de Diego Rosel, cette énumération extravagante réunissant métamorphoses, propos courtisans, pratiques militaires, hiéroglyphes, proses, vers, propos moralisateurs... un inventaire assez étonnant qui attire sinon la curiosité tout au moins le rire, ce rire qui était le propre d'une rhétorique de cour, ce rire courtisan nourri par la culture populaire ${ }^{10}$. Cette notion de rire, encore élargie, sera pratiquée par Cervantès et lui permettra de véhiculer toute la profondeur de sa pensée ${ }^{11}$.

Or ce qui me semble intéressant à retenir est que ces nouvelles variées, ces architectures livresques renvoient à l'idée de collection, à la notion de cabinet de curiosités. Un livre est un cabinet de curiosités virtuel, un meuble constitué de plusieurs tiroirs où nous allons trouver non pas des médailles ou des restes d'animaux, mais des paroles, des phrases et des récits classés, énoncés, ouverts à l'appétit du curieux ${ }^{12}$. Le livre est sans doute la manière la plus simple et la moins onéreuse de prendre part à ce goût de la collection qui émane des couches supérieures.

Les recueils de Cervantès et de Mey présentent des collections à l'état brut, sans aucun cadre narratif, la préface se substituant en quelque sorte à toute modalité de structuration ${ }^{13}$. Diego Rosel, en revanche, opte pour une démarche plus classique, se servant de la cornice chère aux Italiens, qu'il situe pourtant

9. Il s’agit du sonnet intitulé «De Fernando Bermúdez y Carvajal, camarero del duque de Sessa, a Miguel de Cervantes " (Miguel de Cervantes, Novelas ejemplares, I, Edición de Harry Sieber, Madrid, Cátedra, 2003, p. 55).

10. Voir à propos de la notion de rire le livre de José Emilio Burucúa, Corderos y elefantes. La sacralidad y la risa en la modernidad clásica-siglos XV a XVII, Madrid, Miño y Dávila Editores, 2001.

11. Voir à ce propos les travaux d'Augustin Redondo dans Otra manera de leer el "Quijote ". Historia, tradiciones culturales y literatura, Madrid, Castalia, 1998.

12. Sur la notion de collection au XVII ${ }^{e}$ siècle, voir Antoine Schnapper, Le géant, la licorne et la tulipe. Les cabinets de curiosités en France au XVII siècle, Paris, Flammarion, coll. " Champs Arts ", 2012.

13. Sur la préface dans ces recueils de nouvelles voir F. Copello, «La interlocución en prólogos de libros de relatos (1613-1624) », Criticón, n 81-82, 2001, p. 353-367. 
dans un environnement castillan sur les bords du Manzanares ${ }^{14}$, dans une sorte de jardin (image parfaite de l'ensemble du monde, forgée à partir de la notion de paradis). C'est dans cet endroit ameno et de tanta arboleda que vont dialoguer quatre personnages masculins issus de la noblesse. L'échange, porteur d'histoires, va se prolonger tout au long de trois journées qui réunissent au total seize récits ${ }^{15}$ dont huit sont des métamorphoses, textes assez longs, d'une cinquantaine de pages chacun. Dans le résultat final de chaque transformation, nous verrons des animaux ainsi que d'autres éléments produits par la nature ou par l'homme : l'éléphant, l'autruche, le scarabée, la tortue, la taupe, les bois d'ébène et de brésil, le volcan, le caméléon et le camée. Notons au passage que ces mêmes animaux, embaumés, sont souvent présents dans les cabinets de curiosités car ce sont des animaux exotiques; de plus, des morceaux de bois venant d'Afrique ou du Nouveau Monde constituent aussi des curiosités ; par ailleurs, le camée est un bijou "illustré ", ce qui nous rapproche des médailles. Diego Rosel a donc intégré dans son catalogue de métamorphoses des éléments propres à toute collection et les a exposés au regard du lecteur. Les quatre interlocuteurs des Transformaciones sont, à la manière de Philippe $\mathrm{II}^{16}$, des amateurs d'exotisme.

Le livre de Rosel n'est pas, comme on l'a dit, un volume de miscellanées ${ }^{17}$ car tous les récits suivent deux formules possibles bien établies dès le départ et répondent dans leur variété à une cohérence structurelle et thématique. Ces textes correspondent plutôt à la définition qu'en donne José Manuel Pedrosa pour qui il s'agit d'un recueil de contes étiologiques à caractère comique ${ }^{18}$. Comme nous n'allons évoquer ici que les récits intitulés transformaciones (et pas les aplicaciones), nous dirons que cet effet comique provient d'un emploi parodique du genre de la métamorphose ${ }^{19}$. La récriture du texte ovidien par

14. Sur le cadre narratif du livre de Rosel et sur la notion de jardin voir F. Copello, « Marcos narrativos ajardinados en las colecciones de novelas cortas espańolas del siglo XVII ", dans Pierre Civil et Françoise Crémoux (sous la dir. de), Actas del XVI Congreso de la Asociación Internacional de Hispanistas. Nuevos caminos del hispanismo..., Madrid, Iberoamericana-Vervuert, 2010, p. 109-116.

15. Sur la structure du livre voir plus de détails dans F. Copello, « Las Aplicaciones de Diego Rosel y Fuenllana : una reflexión sobre la geografía del relato en la España del siglo XVII ", dans I. Arellano, M. C. Pinillos, F. Serralta et M. Vitse (sous la dir. de), Studia Aurea. Actas del III Congreso de la AISO (Toulouse, 1993). III. Prosa, Toulouse, 1996, p. 129-138; p. 132-133.

16. Voir Fernando Checa Cremades, Felipe II mecenas de las artes, Madrid, Nerea, 1992, passim et en particulier p. 245-247.

17. "Se trata de una extensa miscelánea donde Rosel se ocupa de asuntos diversos ", nous dit José Montero Reguera dans « Humanismo, erudición y parodia en Cervantes : del Quijote al Persiles ", Edad de Oro, n 15, 1996, p. 87-109.

18. Voir José Manuel Pedrosa, "De re etiologica : mitos de orígenes y literatura de la modernidad ", Culturas populares. Revista electrónica, 2, mayo-agosto 2006, et Id. El cuento popular en los Siglos de Oro, Madrid, Arcadia de Letras, 2004, p. 199.

19. Ce qui a fait penser au personnage du Primo dans le Quichotte, à partir de l'analyse d'Alan Soons à laquelle j'adhère. Voir à ce propos des références dans F. Copello, "Las Aplicaciones de Diego Rosel... ", p. 129-130. Le Primo serait un personnage littéraire élaboré à partir de l'identité de Diego Rosel. 
Diego Rosel est, d'une part, un traitement burlesque du genre classique, d'autre part une reprise désopilante de cet autre genre en vogue depuis le XIV siècle : l'Ovide moralisé ${ }^{20}$. Non pas que la réflexion morale soit absente dans le livre en question : on évoque dès le départ l'utilité de ces récits et chacun a un soustitre qui fait référence à un vice ou à une vertu. Citons la Transformation de l'éléphant intitulée aussi Argument de la jalousie, ou celle que nous allons traiter : la Transformation de la tortue (galápago) sous-titrée Argument de la sensualité. Néanmoins, la tonalité prête au rire et dissout dans le ridicule tout propos moralisateur. Nous serions proches, je pense, de ce que Maria Aranda désigne comme " une écriture de l'extravagance (souriante ou grinçante) ${ }^{21}$ destinée à punir par le rire et qui peut passer par la déformation ou la métamorphose. Mais dans le volume de Rosel, publié loin de la métropole, l'effet édifiant est à mon avis complètement absent : on y perçoit même une certaine impertinence littéraire.

Cela dit, il me semble intéressant de remarquer que cet appel au procédé narratif de la métamorphose est l'une des manières d'explorer les possibilités de la fiction. On se rapproche par moments du fantastique, comme le suggère Maria Aranda pour certains textes de María de Zayas et de Cervantès ${ }^{22}$, mais en essayant en même temps de fournir un témoignage sur ce monde qui est en pleine transformation sociale, comme le montre le Quichotte $e^{23}$. Cervantès et Rosel se situent chacun à sa manière dans une mouvance similaire qui essaye de représenter le monde en termes de changement.

Ayant déjà examiné, dans une perspective différente certes, trois récits de Rosel (les métamorphoses de l'éléphant, du scarabée et de la taupe ${ }^{24}$, je vais me concentrer cette fois sur la Transformation de la tortue. Argument de la sensualité qui se trouve être la première de la deuxième journée ${ }^{25}$. Un bref résumé nous dit que cette histoire traite d'un noble chevalier qui se consacre d'abord au commerce, puis aux armes, enfin à ce qui lui plaitt ${ }^{26}$.

Ce récit, long d'une soixantaine de pages, est raconté par Menandro et occupe huit chapitres (XIX à XXVI). Il s'agit d'une histoire itinérante (ce qui permet de varier les épisodes) qui commence dans une ville de Hongrie où le

20. Voir Marylène Possamai-Pérez, L’Ovide moralisé. Essai d'interprétation, Paris, Honoré Champion Éditeur, 2006, ainsi que la bibliographie citée.

21. Maria Aranda, Le spectre en son miroir. Essai sur le texte fantastique au Siècle d'Or, Madrid, Casa de Velázquez, 2011, p. 119.

22. Ibid., voir les pages consacrées à María de Zayas (p. 35-42) et aux Nouvelles exemplaires de Cervantès (p. 54-64) ainsi que les pages préliminaires.

23. Voir mon travail «Fábula y metamorfosis : una variada reflexión sobre el movimiento en el Quijote", dans Juan Diego Vila (sous la dir. de), El "Quijote" desde su contexto cultural, Buenos Aires, EUDEBA, 2013, p. 147-162.

24. F. Copello, " Nouvelle et enfance : l'exemple de Diego Rosel », dans Augustin Redondo (sous la dir. de), Figures de l'enfance, Paris, Publications de la Sorbonne/Presses de la Sorbonne Nouvelle, 1997, p. 191-212.

25. D. Rosel, Parte Primera de varias aplicaciones..., p. 141-204.

26. Ibid., p. 141. 
héros, Torcato, fils unique d'un noble marchand veuf, riche et avare, prend un jour le parti de suivre son empereur et de participer à la guerre. À la mort de son père, il revient chez lui et décide de partir à nouveau et de changer de métier : il devient alchimiste dans une ville somptueuse de la Thrace. Ce sera l'occasion de présenter le premier épisode érotique de l'histoire.

Une dame noble mais pauvre propose à sa plus belle fille, Delia, d'offrir ses charmes à Torcato en échange de cours d'alchimie. Cela permettrait à Delia de devenir riche. Or le dieu Jupiter, vaincu par la beauté de Delia, souhaite vivement coucher avec elle. Il se présente chez Torcato déguisé en homme (il y a déjà dans le récit un certain goût pour la métamorphose) et lui propose de l'aider dans ses projets professionnels puisqu'il est un alchimiste parfait. En échange, Torcato devra favoriser la rencontre entre Jupiter et Delia. Notre héros, plus aimable qu'intéressé, ayant déjà pris Delia « en stage », fait préparer une chambre où Jupiter, au lit, attend Delia dans l'obscurité. La jeune Delia, constatant la méchanceté masculine, croyant qu'il s'agit de Torcato, et non sans plaisir, se laisse faire. Le matin venu, devant la colère de la jeune femme et face à l'embarras de Torcato, Jupiter décide de se faire pardonner : il dévoile son identité et promet d'aider les deux personnages. Par ailleurs, il offre à Delia une bague qui permet de retrouver la virginité. Delia, enchantée et reconnaissante, se demande si par hasard, dans le cas où elle serait tombée enceinte, elle ne deviendrait pas la mère d'un dieu.

Souhaitant chercher de nouveaux horizons, Torcato part une nouvelle fois et décide de devenir marchand de diamants dans une ville importante d'un autre royaume. C'est là qu'il fait la connaissance de l'épouse du corrégidor dont le mari est absent. Séduit par cette femme d'un charme extraordinaire, il passe une nuit d'extase avec elle et, souhaitant retrouver souvent les plaisirs de l'alcôve, accepte de se faire conduire chez la corregidora à l'intérieur d'un clavicorde qui deviendra sa cachette attitrée une fois installé dans la chambre de la bien-aimée, où les amants passent de merveilleuses nuits blanches. Seulement, un jour, le corrégidor revient et découvre la machination : il envisage donc une punition exemplaire.

C'est là, vers la fin du texte, que nous nous retrouvons devant la scène de la transformation. Le corrégidor arrive avec deux coffres et fait introduire chaque victime dans un coffre qui a un trou permettant à chaque coupable de sortir la tête. De plus, grâce à ces trous, l'eau pourra pénétrer dans les caisses lorsqu'elles seront jetées dans le fossé qui entoure le château. La mort de Torcato et de la corregidora est donc sûre et certaine. Cependant, entre temps, l'ancien alchimiste a demandé de l'aide à Jupiter. Celui-ci, pour préserver la vie des victimes, les transforme en des espèces d'animaux encaissés qui ne craignent pas l'eau. Comme le dernier mot prononcé par le corrégidor croyant Torcato bien mort est : "El galán pagó », ces animaux portent le nom de galápagos, c'està-dire de tortues. Le dénouement répond donc aux normes du récit étiologique 
et de la métamorphose. Quelques lignes moralisatrices viennent alors clore l'histoire en évoquant l'importance et la nécessité du mariage ${ }^{27}$.

Le choix de la tortue est sans aucun doute lié à un jeu de mots, mais j'aimerais pousser un peu plus loin la réflexion sur la symbolique de ce reptile assez présent dans la littérature et l'iconographie. Je ne vais m'arrêter dans ce travail que sur ces images qui véhiculent un lien quelconque avec la notion de sensualité.

La tortue est associée à certaines images de la femme, citons pour exemple l'emblème CXCV d'Alciat, intitulé Mulieris famam, non formam vulgatam esse oportere. Le juriste italien considère que la femme honnête, comme la tortue, doit se consacrer aux activités domestiques et rester chez elle. C'est pourquoi l'illustration, qui renvoie à une tradition plus ancienne, montre le portrait de Vénus domestique qui appuie son pied sur une tortue ${ }^{28}$. L'image séduira quelques années plus tard Pedro Soto de Rojas, qui fera construire dans son jardin de Grenade une fontaine reproduisant à l'identique la déesse ménagère et la tortue ${ }^{29}$. Un autre commentaire, plus tardif, d'un personnage de El dia de fiesta por la tarde de Juan de Zabaleta est tout à fait révélateur : "La tortuga, en público, está encerrada. Muy dentro de si ha de estar la mujer en público "30. Il y a donc dans l'horizon d'attente des lecteurs des comparaisons qui associent la tortue à la femme inhibée et pudique. Cela est confirmé par un document plus proche de la période de rédaction des Transformaciones... : la représentation de la Pudicité dans l'Iconologie de Cesare Ripa (dont la première édition illustrée est de 1603), où l'on voit une jeune fille voilée qui foule d'un pied une tortue ${ }^{31}$.

Si la tortue femelle est plutôt associée à la femme interdite de sensualité, la tortue mâle, de son côté, vit une situation assez complexe si l'on en croit Élien (III ${ }^{\mathrm{e}}$ siècle) dans son Histoire naturelle. Pour lui la tortue de terre est un

27. "Quan mal parece las personas ricas y principales, mayormente los que no siguen la guerra, de no tomar estado..." (Ibid., p. 204).

28. Alciato, Emblemas [1531], Edición y comentario : Santiago Sebastián, Prólogo : Aurora Egido, Traducción actualizada de los emblemas : Pilar Pedraza, Madrid, Akal, 1993, p. 239-240.

29. Don Francisco de Trillo Figueroa dans la description du jardin (qui introduit le poème de son ami) évoque cette : "...tortuga de metal que, con cañería sin número y fuerza sin medida, es burlador rostro a rostro de cuantos ocupan las ventanas y escalera... " (Pedro Soto de Rojas, Paraiso cerrado para muchos, jardines abiertos para pocos... [1652], Edición de Aurora Egido, Madrid, Cátedra, 1993, p. 89). Voir aussi les vers de Soto de Rojas (v. 692-699, p. 123) et les commentaires d'Aurora Egido (p. 38).

30. Juan de Zabaleta, El día de fiesta por la mañana y por la tarde [1654 et 1660], Edición, introducción y notas de Cristóbal Cuevas García, Madrid, Castalia, 1983, p. 357.

31. Voir Virginie Bar et Dominique Brême, Dictionnaire iconologique. Les allégories et les symboles de Cesare Ripa et Jean Baudoin, Dijon, Éditions Faton, 1999, p. 325. Voir aussi les commentaires de J. Baudoin sur la Pudicité : «Elle a la teste voilée, pour nous apprendre, qu'une honneste femme doit tenir cachée sa beauté, plustot que d'en faire monstre [...] Quant à la Tortüe qu'elle foule aux pieds, cela veut dire; Que les femmes chastes ne doivent non plus bouger de leur maison [...] qu'une femme de bien ait ses promenades bornées dans l'enclos de son logis " (Iconologie ou explication nouvelle de plusieurs images, emblèmes et autres figures [...] Tirée des Recherches et des Figures de César Ripa, Moralisées par J. Baudoin, Paris, Chez Mathieu Guillemot, 1644, p. 165). 
animal très luxurieux, tout au moins le mâle. Puisque les tortues femelles sont modérément concupiscentes et pensent que la vie vaut mieux que la satisfaction d'un plaisir, les mâles ne peuvent pas les persuader de réaliser l'acte sexuel ${ }^{32}$.

Ces différents textes et documents montrent (et les lecteurs de Rosel ne pouvaient pas être complètement étrangers à ces traditions) que la vie sexuelle à laquelle sont condamnés Torcato et la corregidora risquait d'être extrêmement compliquée $e^{33}$.

Une pensée me vient à propos de quelques lignes de Jacques Derrida dans son L'animal que donc je suis. Le philosophe voit dans l'homme cet animal qui a honte d'être nu comme une bête. Il nous dit : « ...à l'exception de l'homme, aucun animal n'a jamais songé à se vêtir. Le vêtement serait le propre de l'homme, l'un des "propres" de l'homme. Le "se vêtir" serait inséparable de toutes les autres figures du "propre de l'homme" " ${ }^{34}$. Or, la tortue est cet animal emblématique de "l'habillé ", c'est-à-dire cet être qui naturellement cache sa nudité, muni d'une espèce de ceinture de chasteté qui empêche les plaisirs des caresses et de la peau. Torcato et la corregidora, tout en devenant des animaux, sont condamnés à la plus pure urbanité, celle de la honte et de l'habit, celle de la maison qui cache et qui interdit plus qu'elle ne protège.

Voilà le message ludique, extravagant, curieux et, à mon avis, parodique de tout sens moral, de ce récit qui montre l'usage extrêmement libre de la tradition ovidienne chez les hommes du XVII siècle.

32. Voir Xosé Ramón Mariño Ferro, Symboles animaux. Un dictionnaire des représentations et croyances en Occident, Traduit de l'espagnol par Christine Girard et Gérard Grenet, Paris, Desclée de Brouwer, 1996, p. 407 a et b.

33. Dans un texte ancien, les Métamorphoses d'Antoninus Liberalis (circa $\mathrm{II}^{\mathrm{e}}$ siècle), nous voyons Apollon métamorphosé en tortue pour se rapprocher de la belle Dyope, mais il se transforme à nouveau pour pouvoir s'accoupler avec elle (The metamorphoses of Antoninus Liberalis, A translation with a commentary by Francis Celoria, London and New York, Routledge, 1992, p. 91.

34. Jacques Derrida, L'animal que donc je suis, Édition établie par Marie-Louise Mallet, Paris, Galilée, 2006, p. 19. 
